\title{
Variaciones en las manifestaciones clínicas de la giardiosis en pacientes pediátricos hospitalizados, según grupos de edades
}

\author{
Pedro Almirall, Maydel Alfonso, Ivonne Ávila, Yohana Salazar, Angel A. Escobedo, \\ Fidel A. Núñez y Sergio Cimerman
}

\begin{abstract}
Centro Municipal de Higiene, Epidemiología y Microbiología,

La Habana, Cuba. UATS/Plaza (PA)

Universidad de La Habana, La Habana, Cuba.

Facultad Comandante Manue Fajardo (MA)

Hospital Pediátrico

Universitario "Centro Habana",

La Habana, Cuba.

Departamento de Pediatría (IA)

Instituto Nacional de Higiene,

Epidemiología y Microbiología,

La Habana, Cuba.

Departamento de Salud

Escolar (YS)

Hospital Pediátrico

Universitario "Pedro Borrás", La

Habana, Cuba.

Departamento de

Parasitología (AAE)

Instituto "Pedro Kouri", La Habana, Cuba. Departamento de

Parasitología (FAN)

Instituto de Infectologia "Emílio

Ribas". São Paulo, Brasil.

Unidad de Internación (SC).

No existe conflicto de intereses. Fuente de financiamiento: Los salarios del personal de asistencia e investigación fueron asumidos por cada uno de los hospitales instituciones correspondientes a los investigadores.
\end{abstract}

Recibido: 23 de enero de 2013

Aceptado: 15 de julio de 2013

Correspondencia a: Angel A. Escobedo Carbonell escobedo@infomed.sld.cu

\section{Introducción}

G Yiardia lamblia, agente causal de la giardiosis -humana, es el protozoo patógeno que con más frecuencia causa infección intestinal en huma$\operatorname{nos}^{1}$. Aunque fue visto $-\mathrm{y}$ asociado a síntomas- por vez primera por van Leeuwenhoek en 1681, este protozoo fue considerado durante mucho tiempo como comensal. Sin embargo, ya desde los inicios del siglo XX su importancia clínica fue debatida y desde hace algunas décadas es evidente su relación causal con cuadros diarreicos -acompañados o no de malabsorción intestinal-, dolores abdominales, retraso del crecimiento y desarrollo de los niños, así como pobre función cognoscitiva en este mismo grupo $^{2}$. En fecha reciente, la infección por Giardia se ha visto relacionada con fatiga crónica ${ }^{3}$ y síndrome de intestino irritable post-infeccioso $0^{4}$.

La giardiosis ha pasado a ser en gran parte del mundo una enfermedad frecuente y un problema de salud pública. La Organización Mundial de la Salud (OMS) estima que en Asia, África y Latinoamérica existen alrededor de 200 millones de casos, con más de 500.000 casos nuevos cada año. Es así que desde el 2004, debido al impacto de esta parasitosis sobre el desarrollo socio-económico, la OMS la incluyó en la Iniciativa de Enfermedades Desatendidas 5 .
En los países industrializados, esta parasitosis es reconocida como una enfermedad re-emergente, debido a su asociación a numerosos brotes de enfermedad diarreica en guarderías infantiles, a brotes de transmisión hídrica o, en menor medida, por alimentos ${ }^{1,5}$. Todo esto ha focalizado la atención en este protozoo, lo que quizás pueda dar un impulso a las investigaciones relacionadas con este agente parasitario después de años de relativa desatención.

La giardiosis puede ser autolimitada en la mayoría de los casos; sin embargo, debido a la potencial aparición de síntomas intermitentes o crónicos, muchos autores plantean que debe ser tratada siempre y a la mayor brevedad ${ }^{6}$.

Muchas interrogantes científicas acerca de la giardiosis permanecen sin respuesta. Los factores de riesgo y las manifestaciones clínicas en la giardiosis humana han sido ampliamente estudiados en viajeros de países desarrollados procedentes de países donde la enfermedad es altamente endémica o a partir de estudios de brotes epidémicos. Sorpresivamente, es poco el conocimiento acerca de las manifestaciones clínicas de aquellos pacientes hospitalizados en países endémicos; también se desconocen las posibles diferencias que pueden existir en las manifestaciones clínicas, de acuerdo a la edad en los pacientes pediátricos.

Se propone enfocar este estudio en la descripción de las manifestaciones clínicas de los niños hospitalizados 
por giardiosis, comparando los niños bajo 5 años de edad con los de mayor edad.

\section{Pacientes y Métodos}

\section{Diseño y lugar del estudio}

Se realizó un estudio retrospectivo en el Hospital Pediátrico de Centro Habana ubicado en La Habana, Cuba. Este hospital es altamente especializado, cuenta con 226 camas y brinda atención médica a pacientes remitidos de la atención primaria, de otros hospitales pediátricos, así como a pacientes que acuden de manera espontánea. La población infantil que fundamentalmente atiende procede de los municipios Centro Habana, Plaza, Cerro y Habana Vieja. En Cuba y en todo el país existe un adecuado sistema de atención primaria (medicina y enfermería familiar), complementado por los niveles secundarios y terciarios de salud ${ }^{7}$.

\section{Recolección de los datos}

Se solicitó al Departamento de Registros Médicos la lista de todos los pacientes que ingresaron y egresaron entre el $1^{\circ}$ de enero y el 31 de diciembre de 2007 donde la giardiosis estuvo entre los diagnósticos al egreso. Los casos fueron identificados por el código A07-1 y fueron incluidos en el estudio si: sus historias clínicas estaban completas, el diagnóstico al inicio era consistente con giardiosis, y hubo un informe de laboratorio que confirmaba el diagnóstico de quistes y/o trofozoitos de Giardia, a partir de una muestra fecal (por examen directo o método de concentración de formol-éter) o líquido duodenal. Para aquellos pacientes que tuvieron más de un ingreso en el período de estudio, las readmisiones dentro de dos semanas del egreso fueron consideradas como un mismo ingreso; los ingresos repetidos que ocurrieron en un período mayor de dos semanas después del egreso se consideraron como ingresos diferentes.

Las edades agrupadas en $<5$ años $y \geq 5$ años, así como los síntomas y signos, fueron registrados en un formulario prediseñado. Adicionalmente, se extrajo la duración de la estadía hospitalaria.

\section{Análisis estadístico}

Los datos fueron almacenados en Excel y analizados con los paquetes estadísticos EpiInfo 6.04 software (CDC, Atlanta, GA, USA) y GraphPad Prism versión 5.01 para Windows (GraphPad Software, San Diego California USA, www.graphpad.com). Se utilizó la prueba de $\chi^{2}$ para comparar las frecuencias de las manifestaciones clínicas entre los grupos de edades estudiados. Para comparar los días de estadía hospitalaria se utilizó la prueba no paramétrica de Mann Whitney luego de que se verificara que los datos no cumplían la distribución normal. En todos los casos fueron considerados significativos los valores de $\mathrm{p}<$ de 0,05 .

\section{Consideraciones éticas}

El Comité de Ética del Hospital Pediátrico de Centro Habana aprobó el protocolo de investigación. Los datos recolectados de las historias clínicas se manejaron con confidencialidad. La identidad de los pacientes no se incluyó en la base de datos. No se solicitó consentimiento informado debido a que todos los datos analizados habían sido recogidos como parte del procesamiento de rutina de los casos hospitalizados.

\section{Resultados}

\section{Características de los casos y frecuencia por meses}

Un total de 185 pacientes con giardiosis fueron hospitalizados durante el período de estudio. Quince casos no fueron incluidos debido a que las historias clínicas estaban incompletas ( 9 casos) y no estaban a disposición (6 casos). Ciento setenta casos con giardiosis fueron incluidos en el análisis final; de ellos, 97 (57,1\%) eran masculinos. La distribución por grupos de edades fue: $<5$ años, 122 pacientes $(71,8 \%)$ y $\geq 5$ años, $48(28,2 \%)$. Hubo variación en el diagnóstico de la giardiosis por meses observándose las mayores cifras en los meses de enero y febrero (Figura 1). Se comprobó que todos fueron tratados indistintamente con quinacrina, tinidazol, secnidazol o metronidazol.

\section{Manifestaciones clínicas}

Al comparar el grupo de niños bajo 5 años con aquellos de 5 y más años de edad, se encontró que estos últimos manifestaron con una frecuencia mayor el dolor abdominal

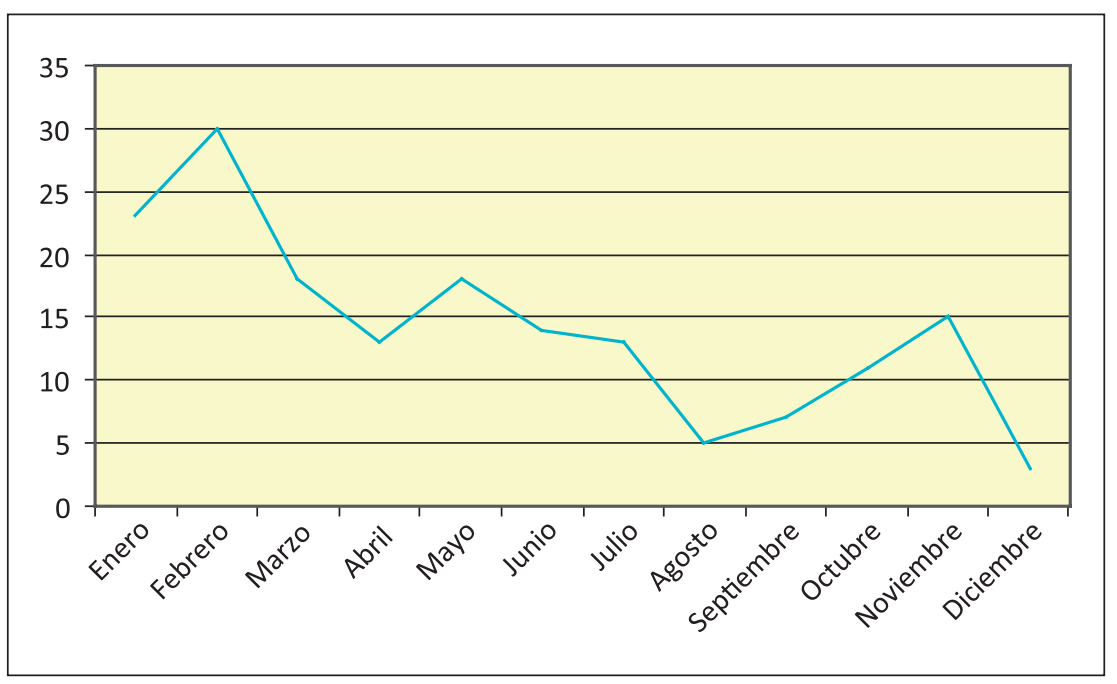

Figura 1. Distribución mensual de la infección por Giardia en los niños hospitalizados en el Hospital Pediátrico "Centro Habana", 2007. Fuente: Encuestas. 
Tabla 1. Comparación de las manifestaciones clínicas entre los niños infectados con Giardia < 5 años, con los $\geq 5$ años ingresados en el Hospital Pediátrico Universitario "Centro Habana". Cuba

\begin{tabular}{|c|c|c|c|c|}
\hline \multirow{2}{*}{$\begin{array}{l}\text { Manifestaciones } \\
\text { clínicas }\end{array}$} & \multicolumn{2}{|c|}{ Grupos de edades } & \multirow[t]{2}{*}{ Valor de $p$} & \multirow[t]{2}{*}{ OR (IC al 95\%) } \\
\hline & $\begin{array}{c}<5 \text { años } \\
(n=122) \\
n \quad(\%)\end{array}$ & $\begin{array}{c}\geq 5 \text { años } \\
(n=48) \\
n \quad(\%)\end{array}$ & & \\
\hline Diarrea & $91(74,6)$ & $15(31,2)$ & $p=0,000+$ & $6,45(3,12-13,37)$ \\
\hline Vómitos & $70(57,4)$ & $22(45,8)$ & $p=0,174$ & $1,59(0,82-3,10)$ \\
\hline Dolor abdominal & $32(26,2)$ & $37(77,1)$ & $p=0,000+$ & $9,46(4,35-20,52)$ \\
\hline Fiebre $\geq 38^{\circ} \mathrm{C}$ & $31(25,4)$ & $8(16,7)$ & $p=0,222$ & $1,70(0,73-3,95)$ \\
\hline Decaimiento & $18(14,7)$ & $7(14,6)$ & $p=0,977$ & $1,01(0,40-2,54)$ \\
\hline Disminución del apetito & $15(12,3)$ & $3(6,2)$ & $p=0,193^{*}$ & $2,10(0,62-7,09)$ \\
\hline Deshidratación & $13(10,6)$ & $7(14,6)$ & $p=0,474$ & $1,43(0,55-3,75)$ \\
\hline Pérdida de peso & $5(4,1)$ & $1(2,1)$ & $p=0,456^{*}$ & $2,01(0,30-13,24)$ \\
\hline Urticaria & $1 \quad(0,8)$ & $4 \quad(8,3)$ & $p=0,023^{*}$ & $11,0 \quad(1,20-101,11)$ \\
\hline
\end{tabular}

*Prueba exacta de Fisher. aquellos niños bajo 6 años de edad y éstos tuvieron un riesgo mayor de estar infectados que los niños entre 6 y 10 años de edad [OR = 3,42; IC al 95\%: 1,29-9,13 $]^{11}$.

Es interesante el hecho que un gran número de pacientes ingresara en dos meses consecutivos (enero y febrero), precisamente en el medio de la temporada de seca (en Cuba es desde noviembre a abril). Los presentes resultados fueron diferentes a aquellos de un estudio previo, de vigilancia de la giardiosis en los E.U.A., realizado entre los años 2003 y 2005, donde se encontró un incremento de la frecuencia de la giardiosis durante los meses de junio a octubre, en niños entre 1 y 14 años de edad ${ }^{12}$. Otro estudio realizado por Naumova y cols., notifica similares resultados al precedente ${ }^{13}$. Es importante tomar en cuenta que en todos los estudios mencionados se analizaron poblaciones abiertas mientras en nuestro grupo de estudio estuvo compuesto sólo por niños diagnosticados por giardiosis y hospitalizados. Entender las fluctuaciones estacionales es un reto, las diferencias muchas veces reflejan diversos factores entre los que se cuentan la heterogeneidad en la susceptibilidad del hospedero, la vía de transmisión, etc.

Algunos estudios han mostrado que las manifestaciones clínicas en niños bajo 5 años con giardiosis pueden variar de aquellos observados en niños mayores ${ }^{14,15}$. Nuestro estudio reafirma la posibilidad de que ciertos síntomas y signos pueden ser característicos de un grupo de edades.

Los síntomas y signos de la giardiosis, especialmente en los niños, son variables y pueden quedar enmascaradas con una gama de alteraciones gastrointestinales infecciosas y no infecciosas. En los pacientes estudiados, prevaleció la diarrea bajo los 5 años de edad, similar a lo observado por Góra-Gebka y cols., quienes encontraron una mayor frecuencia de diarrea crónica bajo 5 años de edad, con relación al grupo de 5 años o más: 20 (90,9\%) vs $4(14,8 \%)$, respectivamente ${ }^{13}$. Esto pudiera estar relacionado con la inmunidad aún inmadura del hospedero en estas edades lo que influiría en sus manifestaciones clínicas $^{16,17}$.

El dolor abdominal fue encontrado más frecuentemente en los niños de 5 y más años de edad. Datos similares se encontraron en un estudio realizado en Rusia ${ }^{15}$. En Polonia, el dolor abdominal crónico también fue encontrado con una mayor frecuencia $(81,5 \%)$ a partir de los 5 años de edad ${ }^{14}$. Es oportuno señalar que en el grupo bajo 5 años están incluidos los lactantes en los cuales es más difícil de registrar este síntoma.

Por lo contrario a lo encontrado en Rusia ${ }^{15}$, en el presente estudio, la urticaria fue fundamentalmente encontrada en los niños sobre 5 años de edad. La posible asociación entre la infección por Giardia y algunas manifestaciones alérgicas ha sido ya discutida por varios autores ${ }^{18,19}$. Cabe señalar que los pacientes relacionados en este estudio estuvieron todos hospitalizados $\mathrm{y}$, por esto, se pudiera inferir 
que son los casos de giardiosis más intensa, entre quienes la urticaria pudiera ser más común. Por otra parte, hay que considerar que para que se produzcan fenómenos alérgicos se requiere sensibilización previa del sistema inmune, lo que ocurre con una mayor probabilidad en los niños mayores $^{20}$. En los pacientes con giardiosis, en general, los daños en la barrera gastro-duodenal pueden incrementar la permeabilidad intestinal ${ }^{21}$ y la posibilidad de urticaria crónica $^{22}$. En ratones inmunocompetentes, pero atímicos, la infección por Giardia causó un incremento significativo de la permeabilidad del intestino delgado que se correlacionó con la colonización de los trofozoitos ${ }^{23}$. En Cuba, altas tasas de prevalencia de la infección por Giardia han sido vistas entre pacientes con urticaria ${ }^{24}$. En el presente estudio, no obstante, la urticaria observada no puede ser inequívocamente atribuida a la infección por Giardia, debido a que la causa de las manifestaciones alérgicas pudieran ocultar otros diagnósticos concomitantes y que no se exploraron adecuadamente.

La duración de la estadía hospitalaria tiene implicaciones para los pacientes y para el sistema de salud; para este último, incluye recursos económicos, cama, trabajadores de la salud y tiempo. La estadía hospitalaria ha sido utilizada como un indicador de eficiencia para el cuidado hospitalario. En este estudio, la estadía hospitalaria fue mayor en el grupo bajo 5 años de edad, similar al un estudio realizado en Escocia ${ }^{25}$. Los niños bajo 5 años quizás tengan mayor probabilidad de experimentar las formas más intensas de la giardiosis, tal vez por su comportamiento a esta edad: falta de continencia fecal, frecuente contacto persona-persona y exploración del mundo por la boca, con la consiguiente succión digital y de objetos, asociado a la inmadurez del sistema inmune y representar su primer contacto con el parásito.

Los hallazgos de este estudio deben ser interpretados tomando en consideración las siguientes limitaciones. Primero, se trata de un estudio retrospectivo de historias clínicas, donde algunos datos pudieron estar ausentes. Segundo, fue un estudio realizado en el escenario hospitalario y la información extraída no entrega datos sobre el perfil clínico de la giardiosis en la comunidad. En consecuencia, los casos estudiados pueden no ser representativos de aquellos que ocurren en la población general y que acuden a los consultorios del médico y la enfermera de familia. De hecho, la mayoría de los casos con giardiosis es diagnosticada en los centros de atención primaria y no son remitidos a los hospitales pediátricos. Los pacientes internados tienden a representar una selecta minoría de todos los pacientes, en quienes la enfermedad es más intensa. En tercer lugar, la identificación de las posibles causas de la diarrea estuvo limitada a los exámenes de laboratorio ordenados por el médico de asistencia. Así, los cultivos de las heces para buscar causa bacteriana no se realizaron sistemáticamente, excepto cuando la causa bacteriana fue altamente sospechosa desde el punto de vista clínico. Tampoco se realizaron estudios virológicos debido a la limitación de recursos. Por lo tanto, es posible que las manifestaciones clínicas en algunos casos pudieran estar contaminadas con infecciones no relacionadas con Giardia. Finalmente, no se hizo seguimiento de los pacientes después del egreso para determinar la posibilidad del desarrollo de morbilidad residual relacionada con la infección por Giardia o las fallas terapéuticas.

A pesar de todo lo anterior, con estos datos se puede profundizar en el cuadro clínico de la giardiosis en pacientes de área endémica y la carga que ocasiona esta enfermedad a los servicios de salud, lo que puede ser de mucha utilidad para pediatras y otros profesionales de la salud con relación al tema del parasitismo intestinal.

Estos resultados nos permiten concluir que las manifestaciones clínicas pueden variar en algunos aspectos entre los diferentes grupos de edades pediátricas. Finalmente, su rápido reconocimiento es esencial con el fin de arribar a un diagnóstico preciso y al tratamiento oportuno.

\section{Resumen}

Introducción: La giardiosis es una enfermedad frecuente a escala internacional. Se ha sugerido por algunos autores que su presentación clínica puede variar con la edad. Objetivo: Comparar las manifestaciones clínicas de la giardiosis en los niños hospitalizados bajo 5 años de edad con las de niños de 5 años o más. Métodos: Estudio retrospectivo realizado en el Hospital Pediátrico de Centro Habana durante el año 2007. Las manifestaciones clínicas fueron extraídas utilizando un formulario prediseñado y separadas de acuerdo a los grupos de edades estudiados. Adicionalmente, se extrajo la estadía hospitalaria. Resultados: Se incluyeron 170 pacientes. Los niños de 5 y más años de edad manifestaron con mayor frecuencia dolor abdominal $(\mathrm{OR}=9,46$; IC 95\%: 4,35-20,52, $\mathrm{p}=0,000+)$ y urticaria $(\mathrm{OR}=11,0$; IC 95\%: 1,205-101,11, p = 0,023), mientras que la diarrea se encontró más frecuentemente bajo 5 años de edad (OR =6,45; IC 95\%: 3,12-13,37, $\mathrm{p}=0,000+)$. En el resto de las manifestaciones clínicas no se encontraron diferencias significativas en sus frecuencias entre ambos grupos de edades $(p>0,05)$. La estadía hospitalaria fue significativamente más prolongada entre los pacientes bajo 5 años de edad. Discusión y conclusiones: Las manifestaciones clínicas pueden variar en algunos aspectos entre los diferentes grupos de edades pediátricas. Su rápido reconocimiento es esencial con el fin de arribar a un diagnóstico preciso y al tratamiento oportuno. 


\section{Referencias bibliográficas}

1.- Escobedo A A, Almirall P, Robertson L J, Mørch K, Franco R M, Hanevik K, et al. Giardiasis: the ever present threat of a neglected disease. Infect Disord Drug Targets 2010; 10: 329-48.

2.- Ajjampur S S, Koshy B, Venkataramani M, Sarkar R, Joseph A A, Jacob K S, et al. Effect of cryptosporidial and giardial diarrhoea on social maturity, intelligence and physical growth in children in a semi-urban slum in south India. Ann Trop Paediatr 2011; 31: 205-12.

3.- Mørch K, Hanevik K, Rortveit G, Wensaas K A, Langeland N. High rate of fatigue and abdominal symptoms 2 years after an outbreak of giardiasis. Trans R Soc Trop Med Hyg 2009; 103: 530-2.

4.- Hanevik K, Dizdar V, Langeland N, Hausken T. Development of functional gastrointestina disorders after Giardia lamblia infection. BMC Gastroenterol 2009; 9: 27.

5.- Savioli L, Smith H, Thompson A. Giardia and Cryptosporidium join the "Neglected Disease Initiative". Trends Parasitol 2006; 22: 203-8.

6.- Robertson L J, Havenik K, Escobedo A A, Mørch K, Langeland N. Giardiasis-why do the symptoms sometimes never stop? Trends Parasitol 2010; 26: 75-82.

7.- Campion E W, Morrissey S. A different modelmedical care in Cuba. N Engl J Med 2013; 368: 297-9.

8.- Rojas L, Núñez F A, Aguiar P H, Silva Ayçaguer L, Álvarez D, Martínez R, et al. Segunda encuesta nacional de infecciones parasitarias intestinales en Cuba, 2009. Rev Cubana Med Trop 2012; 64: 15-21.
9.- Rojas-Rivero L, Núñez-Fernández F A, Robertson L J. Cuban parasitology in review: a revolutionary triumph. Trends Parasitol 2008; 24: 440-8.

10.- Núñez F A, Hernández M, Finlay C M. Longitudinal study of giardiasis in three day care centres of Havana City. Acta Trop 1999; 73: 237-42.

11.- Escobedo A A, Cañete R, Núñez F A. Prevalence, risk factors and clinical features associated with intestinal parasitic infections in children from San Juan y Martínez, Pinar del Río, Cuba. West Indian Med J 2008; 57: 378-81.

12.- Yoder J S, Beach M J. Giardiasis surveillanceUnited States, 2003-2005. MMWR Surveill Summ 2007; 56: 11-8.

13.- Naumova E N, Jagai J S, Matyas B, DeMaría A Jr, MacNeill I B, Griffiths J K. Seasonality in six enterically transmitted diseases and ambient temperature. Epidemiol Infect 2007; 135: 281-92.

14.- Góra-Gebka M, Liberek A, Sikorska-Winiewska G, Jankowska A, Szlagatys-Sidorkiewicz A, Bako W, et al. Giardiasis in children-clinical diversity and diagnostic problems based on own experience. Med Wieku Rozwoj 2006; 10: 529-38.

15.- Zalipaeva T L. Clinical symptoms of Giardia infection in children. Med Parazitol (Mosk) 2002; 3: 29-32.

16.- Ogra P L. Ageing and its possible impact on mucosal immune responses. Ageing Res Rev 2010; 9: 101-6.

17.- Martin R, Nauta A J, Ben Amor K, Knippels L M, Knol J, Garssen J. Early life: gut microbiota and immune development in infancy. Benef Microbes 2010; 1: 367-82.
18.- Prieto-Lastra L, Pérez-Pimiento A, GonzálezSánchez L A, Iglesias-Cadarso A. Urticaria crónica y angioedema en infección por Giardia lamblia. Med Clin (Barc.) 2006; 126: 358-9.

19.- Noenoff P, Domula E, Willing U, Herrmann J. Giardia lamblia-cause of urticaria and pruritus or accidental association? Hautarzt 2006; 57 : 518-20.

20.- Roberts G, Zhang H, Karmaus W, Raza A, Scott M, Matthews S, et al. Trends in cutaneous sensitization in the first 18 years of life: results from the 1989 Isle of Wight birth cohort study. Clin Exp Allergy 2012; 42: 1501-9.

21.- Dagci H, Ustun S, Taner M S, Rezos G, Karacasu F, Budak S. Protozoan infections and intestinal permeability. Acta Trop 2002; 81: 1-5.

22.- Buher S, Reese L, Kuehl F, Lochs H, Zuberbier T. Pseudoallergic reactions in chronic urticaria associated with altered gastroduodenal permeability. Allergy 2004; 59: 1118-23.

23.- Scott K G, Meddings J B, Kirk D R, Lees-Miller S P, Buret A G. Intestinal infection with Giardia spp. reduces epithelial barrier function in a myosin light chain kinasedependent fashion. Gastroenterology 2002; 123: 1179-90.

24.- Martínez-Rodríguez L I, Cantero Zayas L, Alfonso-Fernández L A, Valdés-Sánchez A F, Gómez-Echevarría A H, Ochoa-Ochoa C, et al. Chronic urticaria: humoral and clinical diagnosis of its morphological variables. J Investig Allerg Clin Immunol 1992; 2: 126-30.

25.- Robertson L J. Severe giardiasis and cryptosporidiosis in Scotland, UK. Epidemiol Infect 1996; 117: 551-61. 